\title{
Utilização do método linkage na identificação dos fatores de risco associados à mortalidade infantil: revisão integrativa da literatura
}

Use of the linkage method to identify the risk factors associated with infant mortality: an integrative review of the literature

Sheila Lima Diógenes Santos ${ }^{1}$ Ana Roberta Vilarouca da Silva ${ }^{1}$ Viriato Campelo ${ }^{1}$

Fabiana Teles Rodrigues José Francisco Ribeiro
${ }^{1}$ Programa de PósGraduação em Ciências Saúde, Universidade Federal do Piauí. Av. Frei Serafim 2280, Centro. 64.000-000

Teresina PI Brasil

sheiladiogeness@gmail.com
Abstract Global concern in relation to child mortality gained visibility with the release of the Millennium Development Goals in 2000 in which the reduction of child mortality is included under goal number four, which proposes to reduce the mortality rate of children under five years by two thirds between 1990 and 2015. Reducing child mortality has been a major priority of social health policies of the Brazilian government. The identification of risk factors associated with infant mortality can assist in the planning of actions to restructure and improve care for pregnant women and newborns in order to reduce child mortality. With this in mind, this study sets out to analyze the use of the linkage method to identify risk factors associated with infant mortality. An integrative review of the literature was conducted for this purpose and eight complete articles published between 2008 and 2013 were analyzed using the following key words: infant mortality, risk factors and information systems. The use of the linkage method proved to be very useful, enabling adequate investigation of the factors most strongly related to child mortality, showing its relevance to the study of public health problems.

Key words Infant mortality, Risk factors, Information systems
Resumo A preocupação mundial em relação à mortalidade infantil ganhou visibilidade com a divulgação no ano 2000 dos Objetivos do Desenvolvimento do Milênio, em que sua redução ganha destaque com a meta de número quatro, que propõe diminuir em dois terços sua taxa para crianças menores de cinco anos, no período entre 1990 e 2015. Reduzir a mortalidade infantil tem sido uma das prioridades das políticas sociais de saúde do governo brasileiro. A identificação de fatores de risco relacionados com a mortalidade infantil pode auxiliar no planejamento de ações para a reestruturação e melhoria da assistência à gestante e aos recém-nascidos, visando à redução da mortalidade infantil. Nesta perspectiva, o presente estudo tem como objetivo analisar a utilização do método de linkage na identificação de fatores de risco associados à mortalidade infantil. Utilizou-se a Revisão Integrativa da Literatura. Foram analisados oito artigos na integra publicados entre 2008 e 2013, através dos descritores: mortalidade infantil, fatores de risco e sistemas de informação. O uso da técnica de linkage mostrou-se bastante útil, permitindo a adequada investigação dos fatores mais fortemente relacionados à mortalidade infantil, mostrando sua relevância para o estudo de problemas de saúde pública. Palavras-chave Mortalidade infantil, Fatores de risco, Sistemas de informação 


\section{Introdução}

A preocupação mundial em relação à mortalidade infantil ganhou visibilidade com a divulgação no ano 2000 dos Objetivos do Desenvolvimento do Milênio, em que a redução da mortalidade infantil ganha destaque com a meta que propõe reduzir em dois terços a taxa de mortalidade de crianças menores de cinco anos, no período entre 1990 e 2015, além de representar ponto crítico para realização de outras relacionadas ao bemestar, à equidade e à redução da pobreza. $\mathrm{O}$ Brasil é um dos signatários desses objetivos ${ }^{1}$.

Reduzir a mortalidade infantil tem sido uma das principais prioridades das políticas sociais de saúde do governo brasileiro por várias décadas. Com a criação do Sistema Único de Saúde (SUS), ocorreram melhorias no acesso aos serviços de saúde e da qualidade dos cuidados de saúde no Brasil, principalmente para a parte da população mais pobre. Mais investimentos em recursos humanos, ciência e tecnologia e cuidados primários à saúde implicaram em maior organização do atendimento às gestantes e recém-nascidos. Entre 1990 e 2007 houve queda importante na mortalidade infantil em todas as regiões do Brasil, especialmente entre a população mais pobre, devido à melhoria das condições socioeconômicas, de infraestrutura urbana e de saúde ${ }^{2,3}$.

Sabe-se que com a melhora na estrutura da assistência perinatal muitos óbitos infantis poderiam ser evitados. Nesse contexto, o Ministério as Saúde implantou uma estratégia em 2011, criando uma rede de atenção que garante acesso e resolutividade durante o pré-natal, o parto e o período neonatal, a Rede Cegonha. Esta, por sua vez, teria maior efetividade em cada região se apoiada em estudo epidemiológico recente sobre os fatores de risco da mortalidade infantil ${ }^{4}$. Além do papel determinante dos serviços de saúde, os fatores socioeconômicos também geram uma importante influência nos casos de mortalidade infantil ${ }^{5}$.

Diante do exposto, sabe-se que no Brasil existe um alto grau de heterogeneidade entre as regiões brasileiras, provocadas por acentuadas diferenças socioeconômicas e culturais, além das iniquidades de acesso aos serviços de saúde, sendo relevante a investigação de fatores de risco locais ${ }^{5}$. $\mathrm{O}$ estudo dos fatores de risco envolvidos na cadeia causal de mortalidade infantil é fundamental para a compreensão das condições de vida da população materno-infantil e para a definição de ações prioritárias de políticas públicas apropriadas a cada realidade e na formulação de es- tratégias que permitam o seu controle e a organização da rede assistencial ${ }^{6,7}$.

A identificação de fatores de risco relacionados com a mortalidade infantil pode auxiliar no planejamento de ações para a reestruturação e a melhoria da assistência à gestante e aos recémnascidos, visando à redução da mortalidade infantil. Essa redução não depende de novos conhecimentos, como ocorre com outros problemas de saúde, mas de garantia da acessibilidade e da utilização mais efetiva do conhecimento científico e tecnológico já existente ${ }^{3,4,8}$.

Dados secundários de Sistemas de Informações em Saúde (SIS) oficiais do Brasil têm sido amplamente utilizados para monitorar e dimensionar os fatores de risco envolvidos na mortalidade infantil. O uso de sistemas de informações para a realização de estudos epidemiológicos depende do grau de cobertura do evento em questão, da integridade e dos detalhes das variáveis nesses sistemas, e da validade de seus dados ${ }^{2}$.

A aplicação da metodologia de relacionamento de bases de dados ou linkage, uma opção de relativo baixo custo operacional, com excelente viabilidade, tem como vantagem a possibilidade de análises mais detalhadas da qualidade de dados oficiais e também com o ganho de informações comuns aos sistemas pareados. No estudo da mortalidade infantil esta metodologia tem sido empregada e se mostrado uma ferramenta útil ${ }^{6,8}$.

Nesta perspectiva, o presente estudo tem como objetivo geral analisar a utilização do método de linkage na identificação de fatores de risco associados à mortalidade infantil. $\mathrm{E}$ como objetivos específicos: identificar o delineamento de pesquisa e as análises estatísticas utilizadas; determinar o percentual de vinculação entre banco de dados do Sistema de Informações sobre Mortalidade (SIM) e Sistema de Informação sobre Nascidos Vivos (SINASC); avaliar se o linkage se mostrou um método viável nos estudos de mortalidade infantil.

\section{Métodos}

Utilizou-se a Revisão Integrativa da Literatura, um método de pesquisa que permite a busca, a avaliação crítica e a síntese das evidências disponíveis do tema investigado, sendo o seu produto final o estado atual do conhecimento do tema investigado, possibilitando ao leitor a acesso a diversas pesquisas realizadas, em um único estudo?.

A questão norteadora da presente investigação foi: o relacionamento de banco de dados ou 
método de linkage é um método adequado na identificação de fatores de risco associados à mortalidade infantil?

Para realizar o levantamento bibliográfico foram selecionados os descritores disponíveis na lista Health Science Descriptors/Medical Subject Headings (DeCS/MeSH), a saber: "mortalidade infantil", "fatores de risco" e "sistemas de informação" e seus equivalentes nos idiomas inglês. Esses descritores foram utilizados para busca nas seguintes bases de dados bibliográficos: Sistema Latino-Americano e do Caribe de Informação em Ciências da Saúde (Lilacs), Scientific Electronic Library Online (Scielo) e Medical Literature Analysis and Retrieval System Online (Medline).

Para busca foram considerados os seguintes parâmetros limitadores: artigos publicados entre 2008 e 2013; redigidos em língua portuguesa, espanhola ou inglesa; e, publicações que tivessem como foco o pareamento entre o SIM e o SINASC para a identificação dos fatores de risco associados à mortalidade infantil. Foram excluídas as publicações que não estivessem em formato de artigo científico, como livros, teses, dissertações, resenhas, cartas e editoriais, ou que não estavam disponíveis na íntegra para acesso online. Foram excluídos, ainda, os artigos cujo objetivo geral fosse o de avaliar a confiabilidade e a completitude dos Sistemas de Informações em Saúde; estudos identificando a ocorrência do pareamento com outros sistemas de informações além dos já citados; e também aqueles em que houvesse a presença de outra etapa na metodologia além do pareamento de banco de dados.

O corpus desta revisão terminou constituído de oito artigos qualificados, após a aplicação dos critérios de inclusão e exclusão e o refinamento posterior da busca. A análise dos artigos ocorreu de forma descritiva, de acordo com o instrumento de coleta de dados, conforme Quadro 1, o qual apresenta a caracterização geral dos artigos selecionados de acordo com título, instituição do autor principal, ano de publicação, periódico publicado e desenho do estudo. Os resultados foram sistematizados permitindo avaliar as seguintes características de cada artigo: dados referentes à publicação (periódico e ano); instituição de origem dos autores principais; delineamento da pesquisa; objetivos e população do estudo; metodologia e análises estatísticas empregadas; resultados e contribuições do estudo.

\section{Resultados}

Foram analisados oito artigos na íntegra e após elaboração do Quadro 1, que apresenta a caracterização geral dos mesmos, observou-se o predomínio de pesquisas em anos mais recentes, encontrando-se quatro publicados no ano de 2012. Em relação à instituição de origem dos autores principais, seis são vinculados a universidades e dois a fundações de pesquisas. Houve predomínio de publicações em revista na área de saúde pública (Caderno de Saúde Pública) com três artigos, seguido por dois na de epidemiologia, dois na de enfermagem e um na de medicina-pediatria.

Notou-se certa equivalência com relação ao delineamento de pesquisa, sendo que o tipo coorte e o tipo caso-controle apareceram em três estudos cada um deles, houve um estudo tipo transversal e outro tipo exploratório, conforme desenhos de metodologia determinados pelos autores. As populações de estudo dos artigos pesquisados encontram-se apresentadas no Quadro 2.

A totalidade dos artigos tinha como objetivo identificar os fatores de risco associados à mortalidade infantil, por meio do pareamento ou linkage entre o SIM e o SINASC.

No estudo realizado em Londrina (PR) foram identificados os fatores de risco em dois períodos distintos, sendo realizada a comparação entre ambos e observada a modificação nesses fatores com o decorrer do tempo e a implementação de políticas públicas brasileiras ${ }^{2}$. Em outro estudo, publicado no ano de 2012, foram identificados os fatores de risco em cinco cidades brasileiras, podendo ser apresentados seus diferenciais em cada local abordado ${ }^{6}$. Na cidade de Reci$\mathrm{fe}$, os fatores de risco foram apresentados sob a perspectiva da evitabilidade ${ }^{10}$.

Para a análise estatística a quase totalidade dos estudos utilizou como medida de associação o odds ratio ( $\mathrm{OR})$ e fez análise bivariada e de regressão logística pelo modelo conceitual hierárquico. O uso de uma abordagem hierarquizada para análise das associações mostrou-se como forma conceitualmente coerente de detectar esta estrutura de determinação do desfecho, mantendo no modelo final as variáveis explicativas baseando-se não apenas no critério da significância estatística. 
Quadro 1. Caracterização geral dos artigos selecionados de acordo com título, instituição do autor principal, ano de publicação, periódico publicado e desenho do estudo.

\begin{tabular}{|c|c|c|c|c|c|}
\hline & Títulos dos artigos & $\begin{array}{c}\text { Instituição autor } \\
\text { principal }\end{array}$ & $\begin{array}{c}\text { Ano } \\
\text { publicação }\end{array}$ & Periódico & $\begin{array}{l}\text { Desenho } \\
\text { do estudo }\end{array}$ \\
\hline 1 & $\begin{array}{l}\text { Risk factors for infant } \\
\text { mortality in a municipality in } \\
\text { southern Brazil: a comparison } \\
\text { of two cohorts using } \\
\text { hierarchical analysis }{ }^{2} \text {. }\end{array}$ & $\begin{array}{l}\text { Programa de Pós- } \\
\text { graduação em Saúde } \\
\text { Coletiva, Universidade } \\
\text { Estadual de Londrina, } \\
\text { Londrina, Brasil. }\end{array}$ & 2012 & $\begin{array}{l}\text { Cad Saude } \\
\text { Publica }\end{array}$ & Coorte \\
\hline 2 & $\begin{array}{l}\text { Diferenciais nos fatores de } \\
\text { risco para a mortalidade } \\
\text { infantil em cinco cidades } \\
\text { brasileiras: um estudo de } \\
\text { caso-controle com base no } \\
\text { SIM e no SINASC }\end{array}$ & $\begin{array}{l}\text { Centro de Pesquisas } \\
\text { Aggeu Magalhães, } \\
\text { Fundação Oswaldo } \\
\text { Cruz, Recife, Brasil. }\end{array}$ & 2012 & $\begin{array}{l}\text { Cad Saude } \\
\text { Publica }\end{array}$ & Caso-controle \\
\hline 3 & $\begin{array}{l}\text { Fatores de risco para } \\
\text { mortalidade neonatal no } \\
\text { município de Serra, Espírito } \\
\text { Santo }^{8} \text {. }\end{array}$ & $\begin{array}{l}\text { Universidade Federal } \\
\text { do Espírito Santo, } \\
\text { Vitória, Espírito Santo, } \\
\text { Brasil. }\end{array}$ & 2012 & $\begin{array}{l}\text { Rev. Bras. } \\
\text { Enferm }\end{array}$ & Coorte \\
\hline 4 & $\begin{array}{l}\text { Fatores de risco para } \\
\text { mortalidade infantil em } \\
\text { municípios do Estado do } \\
\text { Paraná, de } 1997 \text { a } 2008^{7} .\end{array}$ & $\begin{array}{l}\text { Universidade Estadual } \\
\text { de Maringá, Maringá, } \\
\text { Paraná, Brasil. }\end{array}$ & 2012 & $\begin{array}{l}\text { Rev. Paul. } \\
\text { Pediatr }\end{array}$ & Coorte \\
\hline 5 & $\begin{array}{l}\text { Mortalidade neonatal e } \\
\text { evitabilidade: uma análise do } \\
\text { perfil epidemiológico }{ }^{10} \text {. }\end{array}$ & $\begin{array}{l}\text { Fundação Joaquim } \\
\text { Nabuco. Recife, } \\
\text { Pernambuco, Brasil. }\end{array}$ & 2011 & $\begin{array}{l}\text { Rev. Enferm. } \\
\text { UERJ }\end{array}$ & Transversal \\
\hline 6 & $\begin{array}{l}\text { Estudo de fatores de risco } \\
\text { para óbitos de menores de um } \\
\text { ano mediante } \\
\text { compartilhamento de bancos } \\
\text { de dados }{ }^{11} \text {. }\end{array}$ & $\begin{array}{l}\text { Secretaria de Saúde do } \\
\text { Estado da Bahia, } \\
\text { Salvador, Brasil. Instituto } \\
\text { de Saúde Coletiva, } \\
\text { Universidade Federal da } \\
\text { Bahia, Salvador, Brasil. }\end{array}$ & 2008 & $\begin{array}{l}\text { Cad Saude } \\
\text { Publica }\end{array}$ & Caso-controle \\
\hline 7 & $\begin{array}{l}\text { Fatores associados à } \\
\text { mortalidade neonatal precoce: } \\
\text { análise de situação no nível } \\
\text { local }^{12} \text {. }\end{array}$ & $\begin{array}{l}\text { Secretaria Municipal de } \\
\text { Saúde, Salvador, Bahia, } \\
\text { Brasil.Instituto de Saúde } \\
\text { Coletiva, Universidade } \\
\text { Federal da Bahia, } \\
\text { Salvador, Bahia, Brasil. }\end{array}$ & 2010 & $\begin{array}{l}\text { Epidemiol. } \\
\text { Serv. Saúde }\end{array}$ & Exploratório \\
\hline 8 & $\begin{array}{l}\text { Fatores associados à } \\
\text { mortalidade infantil no } \\
\text { Município de Foz do Iguaçu, } \\
\text { Paraná, Brasil: estudo de } \\
\text { caso-controle }^{13} \text {. }\end{array}$ & $\begin{array}{l}\text { Mestrado } \\
\text { Profissionalizante de } \\
\text { Saúde Pública Baseada } \\
\text { em Evidências, Centro } \\
\text { de Pesquisas } \\
\text { Epidemiológicas, } \\
\text { Universidade Federal de } \\
\text { Pelotas, Rio Grande do } \\
\text { Sul, Brasil. }\end{array}$ & 2011 & $\begin{array}{l}\text { Epidemiol. } \\
\text { Serv. Saúde }\end{array}$ & Caso-controle \\
\hline
\end{tabular}

Sistema de Informações sobre Mortalidade - SIM; Sistema de Informação sobre Nascidos Vivos - SINASC. 
Quadro 2. Populações de estudo dos artigos pesquisados.

\begin{tabular}{|c|c|c|}
\hline & Títulos dos artigos & Populações de estudo \\
\hline 1 & $\begin{array}{l}\text { Risk factors for infant mortality in a } \\
\text { municipality in southern Brazil: a comparison } \\
\text { of two cohorts using hierarchical analysis }{ }^{2} \text {. }\end{array}$ & $\begin{array}{l}2 \text { coortes retrospectivas de nascidos vivos de } \\
\text { Londrina, Paraná, Brasil (2000-2001; 2007- } \\
\text { 2008). }\end{array}$ \\
\hline 2 & $\begin{array}{l}\text { Diferenciais nos fatores de risco para a } \\
\text { mortalidade infantil em cinco cidades } \\
\text { brasileiras: um estudo de caso-controle com } \\
\text { base no SIM e no SINASC }{ }^{6} \text {. }\end{array}$ & $\begin{array}{l}1 \text { caso para cada } 3 \text { controles de óbitos infantis das } \\
\text { cidades de Belém, Recife, Guarulhos, Porto Alegre } \\
\text { e Goiânia. }\end{array}$ \\
\hline 3 & $\begin{array}{l}\text { Fatores de risco para mortalidade neonatal no } \\
\text { município de Serra, Espírito Santo }{ }^{8} \text {. }\end{array}$ & $\begin{array}{l}\text { Coorte retrospectiva no município de Serra, } \\
\text { Espírito Santo, entre } 01 / 01 / 2001 \text { a } 31 / 12 / 2005 \text {. }\end{array}$ \\
\hline 4 & $\begin{array}{l}\text { Fatores de risco para mortalidade infantil em } \\
\text { municípios do Estado do Paraná, de } 1997 \text { a } \\
2008^{7} \text {. }\end{array}$ & $\begin{array}{l}\text { Coorte retrospectiva de nascidos vivos e dos } \\
\text { respectivos óbitos de menores de um ano de } \\
\text { idade, filhos de mães residentes nos municípios } \\
\text { que compõem a 9a Regional de Saúde do Paraná, } \\
\text { no período de } 1997 \text { a 2008, divididos em } 4 \\
\text { triênios. }\end{array}$ \\
\hline 5 & $\begin{array}{l}\text { Mortalidade neonatal e evitabilidade: uma } \\
\text { análise do perfil epidemiológico }{ }^{11} \text {. }\end{array}$ & $\begin{array}{l}\text { Óbitos neonatais originados do conjunto de } \\
\text { nascidos vivos de mães residentes emRecife, no } \\
\text { período de } 1999 \text { a } 2009 \text {. }\end{array}$ \\
\hline 6 & $\begin{array}{l}\text { Estudo de fatores de risco para óbitos de } \\
\text { menores de um ano mediante } \\
\text { compartilhamento de bancos de dados }{ }^{12} \text {. }\end{array}$ & $\begin{array}{l}1 \text { caso para cada } 2 \text { controles abrangendo todos os } \\
\text { nascidos vivos do ano 2000, cujas mães residiam } \\
\text { em Salvador, Bahia. }\end{array}$ \\
\hline 7 & $\begin{array}{l}\text { Fatores associados à mortalidade neonatal } \\
\text { precoce: análise de situação no nível local }{ }^{13} \text {. }\end{array}$ & $\begin{array}{l}\text { Nascidos vivos e óbitos infantis ocorridos no } \\
\text { período de } 2000 \text { a 2005, no município de } \\
\text { Salvador, Bahia. }\end{array}$ \\
\hline 8 & $\begin{array}{l}\text { Fatores associados à mortalidade infantil no } \\
\text { Município de Foz do Iguaçu, Paraná, Brasil: } \\
\text { estudo de caso-controle }{ }^{14} \text {. }\end{array}$ & $\begin{array}{l}\text { Crianças nascidas no município de Foz do Iguaçu } \\
\text { que foram a óbito no próprio município ano de } \\
2007 \text { antes de completarem um ano de idade. }\end{array}$ \\
\hline
\end{tabular}

Sistema de Informações sobre Mortalidade - SIM; Sistema de Informação sobre Nascidos Vivos - SINASC.

Apenas o estudo na cidade de Recife utilizou a estatística descritiva com distribuição de frequências (absoluta e relativa) e medidas de tendência central e dispersão ${ }^{14}$.

Nos resultados identificados nos artigos, em dois estudos realizados no estado do Paraná, um na 9a Regional de Saúde do Paraná, outro na cidade de Foz do Iguaçu, não mencionaram o percentual de pareamento ou vinculação entre o SIM e o SINASC, porém afirmaram que a qualidade e cobertura dos dados dos municípios apresentaram-se satisfatórios para execução do estudo com o linkage ${ }^{7,14}$.

$\mathrm{Na}$ cidade de Londrina (PR) foi possível vincular $97,3 \%$ no período de $2000-2001$ e $100 \%$ no período de 2007-2008. No estudo que comparou 5 cidades brasileiras encontrou-se uma média de 90\% de pareamento, em Porto Alegre chegou a $96,70 \%$ e em Belém, para o mesmo ano, somente $81,60 \%$, demonstrando desigualdades regionais na qualidade dos bancos de dados trabalhados. Das declarações de óbito, 90,69\% e 93,20\% foram vinculadas às suas respectivas declarações de nascido vivo nas cidades de Serra e de Recife, respectivamente. $\mathrm{Na}$ cidade de Salvador foram encontrados dois estudos, que apresentaram valores próximos de percentual de pareamento, $73,40 \%$ e $72,42 \%$.

No estudo realizado em Londrina (PR) foram observadas mudanças no perfil de risco para 
a mortalidade infantil entre os dois períodos estudados, devendo estar relacionadas com a expansão das políticas sociais e de cuidados de saúde primários e as mudanças nos padrões reprodutivos e social das mulheres brasileiras. Os autores evidenciaram que o uso dos SIS depende do grau de cobertura, integridade e validade dos dados. Tendo-se em Londrina observado uma melhora da qualidade SINASC, ao tempo em que o linkage ajudou a aprimorar esses dados. Como limitação citaram algumas variáveis importantes não incluídas, como violência materna, tabagismo e doenças maternas².

A pesquisa envolvendo cinco cidades brasileiras revelou marcantes desigualdades no perfil da mortalidade infantil. A seleção das cinco cidades, sendo uma de cada macrorregião brasileira, foi uma alternativa que os autores utilizaram para apreender as diferenças regionais, embora não representando toda a complexidade dessa questão, os achados apontaram para importantes diferenciais regionais entre os fatores de risco para a mortalidade infantil. Revelaram também que apesar da maior determinação da mortalidade infantil pelos fatores biológicos, destacouse a importância da condição socioeconômica e da assistência pública à saúde da gestante e do recém-nascido como determinantes, uma vez que constituem fatores passíveis de mudança garantidas por políticas públicas de qualidade e um crescimento social da população ${ }^{6}$.

Os autores que investigaram o município de Serra (ES) afirmaram que o uso de dados secundários não foi um fator limitante, pois, o estudo apresentou uma baixa perda de dados que pode estar relacionada à boa qualidade de preenchimento das Declarações de Nascido Vivo (DNV) e das Declarações de Óbito (DO). Além de afirmarem que as pesquisas que utilizam o procedimento de linkage de bancos de dados de nascidos vivos e óbitos infantis mostraram a viabilidade e as vantagens dessa estratégia, utilizando-se como fonte de dados os sistemas de informações em saúde disponíveis no Brasil. Essa estratégia de análise possibilitou o monitoramento dos fatores de risco para mortalidade infantil e das mudanças na magnitude desses fatores nas coortes de nascidos vivos. O estudo recomendou essa estratégia para o monitoramento e a identificação dos principais fatores de risco para a mortalidade neonatal e reafirmou a importância da relação dos fatores biológicos com o óbito ${ }^{8}$.

No estudo com os municípios da $9^{a}$ Regional de Saúde, evidenciou-se a necessidade de vigilância contínua em relação à oferta de assistência adequada ao subgrupo de recém-nascidos com maior risco de mortalidade e também a necessidade de que os gestores envidem esforços para garantir assistência pré-natal de qualidade para a população em geral e, de forma particular, para as mulheres com características sociodemográficas mais desfavoráveis. Como também a capacitação dos profissionais da área da saúde, da atenção interdisciplinar para um diagnóstico precoce da vulnerabilidade da criança e do acionamento de medidas que evitem a ocorrência do óbito. A redução da mortalidade infantil nos municípios em estudo pode estar associada à existência dos Comitês Municipais de Mortalidade Infantil e à melhoria da qualidade da atenção básica e ampliação da Estratégia Saúde da Família ${ }^{7}$.

Em Recife (PE), 80\% dos óbitos foram considerados evitáveis. A partir do pareamento do banco de dados de nascimentos e óbitos foi possível identificar os recém-nascidos de risco, além de avaliar as variáveis relacionadas ao pré-natal e ao parto. A técnica de linkage demonstrou sua potencialidade para o estudo da mortalidade infantil, sendo rápida e fácil de ser utilizada, podendo ser empregada na rotina dos serviços de vigilância do óbito infantil ${ }^{10}$.

$\mathrm{Na}$ estudo envolvendo a cidade de Salvador (BA), com dados do ano de 2000, os resultados alertaram para um baixo percentual de preenchimento da maioria dos campos referentes a óbitos de menores de um ano disponibilizados pelo SIM, além de incorreções na classificação destes, como fetal ou não fetal, identificados durante o processo de linkage, que podem se constituir como fontes de erro quando se faz uso exclusivo do SIM como fonte de dados para estudos sobre mortalidade infantil. O SINASC também apresentou omissão de preenchimento de alguns campos importantes, a exemplo de ocupação materna, índice Apgar, filhos tidos e raça/cor. Todavia, observou-se bom nível de coincidência de dados após realização do linkage, contemplando maior gama de dados acerca dos principais fatores de risco para óbitos de menores de um ano ${ }^{12}$.

Já em outro estudo realizado também em Salvador, no período entre os anos 2000 a 2005, mostrou-se que as características sociodemográficas maternas, reprodutivas, assistenciais e do recém-nascido são importantes indicadores que podem ser utilizados como condições de alerta para monitoramento da mortalidade infantil. $\mathrm{O}$ uso da técnica de linkage entre os bancos de dados SIM e SINASC mostrou-se bastante útil, permitindo a adequada investigação dos fatores 
mais fortemente relacionados à mortalidade infantil, mostrando a relevância desses sistemas de informações para o estudo de problemas de saúde. As taxas de cobertura dos sistemas analisados permitiram a produção de dados que representaram a realidade local ${ }^{13}$.

$\mathrm{Na}$ pesquisa feita em Foz do Iguaçu (PR), revelaram-se importantes fatores de risco envolvidos na assistência ao recém-nascido. A redução da mortalidade infantil constitui o maior desafio para os gestores de saúde, principalmente no seu componente neonatal. Sabendo-se, excluídas as anomalias congênitas, que as demais variáveis estão intrinsecamente relacionadas à oferta de serviços de saúde de qualidade, principalmente pelo programa de pré-natal e assistência ao parto, para a melhoria das condições de vida da população geral ${ }^{14}$.

\section{Discussão}

Em várias regiões do mundo a mortalidade infantil vem diminuindo. A diminuição média anual da taxa de mortalidade infantil (TMI) no Brasil foi de $4,8 \%$ ao ano, entre 1990 e 2007 , tendo o componente pós-neonatal apresentado maior tendência de queda com 7,3\% ao ano que o componente neonatal precoce, que teve queda de $3,1 \%$ ao ano. A partir da década de 90 , em virtude da redução do componente pós-neonatal, aumentou o peso relativo às mortes ocorridas no período neonatal ${ }^{3,7}$. O componente neonatal tem se apresentado como principal, que apesar de também apresentar tendência de queda, ainda se mantém em níveis elevados no Brasil, constituindo-se como uma crescente preocupação em saúde pública ${ }^{5}$.

Existem diferenças regionais e desigualdades entre os índices de mortalidade infantil nos distintos estratos sociais no Brasil. Em 2000 a TMI entre os $20 \%$ mais pobres equivalia a mais que o dobro da taxa identificada entre os $20 \%$ mais ricos. As desigualdades regionais ainda são as que mantêm a TMI do Brasil em maior proporção do que outros países da América Latina ${ }^{11}$.

Nos países desenvolvidos a mortalidade neonatal é geralmente determinada por malformações congênitas, enquanto que nos países em desenvolvimento está relacionada principalmente aos diversos fatores de risco relacionados às condições gerais de vida e acesso aos serviços de saú$\mathrm{de}^{8}$. O componente pós-neonatal, nos países desenvolvidos, é pouco significativo, porém no Brasil, apesar do decréscimo nos últimos anos, ain- da apresenta níveis elevados, eticamente inaceitáveis. Sendo em sua maioria relacionado às causas passíveis de redução, principalmente nos grupos sociais que evidenciam piores condições de vida e de acesso dos serviços de saúde ${ }^{11}$.

Dados secundários de Sistemas de Informações em Saúde oficiais do Brasil têm sido amplamente utilizados para monitorar e dimensionar os fatores de risco envolvidos na mortalidade infantil. O uso de sistemas de informações para a realização de estudos epidemiológicos depende do grau de cobertura do evento em questão, da integridade e dos detalhes das variáveis nesses sistemas, e da validade de seus dados ${ }^{2}$.

Em 1975, o Ministério da Saúde promoveu a implantação de um sistema nacional de vigilância epidemiológica e de um modelo único de declaração de óbito, o Sistema de Informações sobre Mortalidade (SIM) ${ }^{15}$.

O reconhecimento da importância das informações sobre nascimentos vivos para as estatísticas de saúde, epidemiologia e demografia, levou o Ministério da Saúde do Brasil a implantar, em 1990, o Sistema de Informação sobre Nascidos Vivos (SINASC). Sendo este de âmbito nacional e sob a responsabilidade das Secretarias Municipais e Estaduais de Saúde. Foi implantado com o objetivo principal de conhecer o perfil epidemiológico dos nascimentos vivos, agregando informações sobre as mães, a gravidez, o parto e o recém-nascido com um panorama epidemiológico mais detalhado e espacialmente desagregado das condições de saúde materno-infantil. Permitindo o acompanhamento do perfil deste segmento da população para traçar políticas de saúde adequadas voltadas para grupos específicos, de acordo com o perfil de risco ${ }^{6,8,16}$.

O SINASC baseia-se nos dados contidos na Declaração de Nascido Vivo (DNV), documento oficial e padronizado para todo o país. Sendo de emissão obrigatória nos hospitais e em outras instituições de saúde nas quais são realizados partos. E nos Cartórios de Registro Civil para os partos realizados no domicílio. Estudos científicos baseados em dados secundários possuem a desvantagem de viés de informação. Entretanto, têm como vantagem a realização a baixo custo de estudos de base populacional e de abrangência nacional. Dessa forma, o SINASC torna-se uma ferramenta essencial para o conhecimento do perfil epidemiológico, o planejamento e a avaliação das ações de saúde materno-infantil. Temse observado progressivo aperfeiçoamento do SIM e do SINASC com um crescente nível de cobertura e a melhora da qualidade dos dados ${ }^{6,16,17}$. 
A aplicação da metodologia de relacionamento de bases de dados ou linkage é uma opção de relativo baixo custo operacional, com excelente viabilidade, que tem como vantagem a possibilidade de análises mais detalhadas da qualidade de dados oficiais e ganho na quantidade de informações comuns aos sistemas relacionados. Nos estudos sobre mortalidade infantil esta metodologia tem sido empregada e se mostrado uma ferramenta útil, permitindo melhorar os dados gravados no SIM e SINASC, tanto em relação à confiabilidade como à completitude ${ }^{2,6,8,16}$.

O linkage deveria ser uma das medidas a ser adotada na rotina dos serviços municipais de saúde, e a gestão poderia incentivar a utilizá-lo para o relacionamento dos seus bancos de dados consolidados. Ressalta-se a possibilidade do uso do linkage como uma importante estratégia de aprimoramento da qualidade dos sistemas nacionais de informações em saúde, identificando e resgatando informações que estão presentes em um sistema e ausentes ou incompletas no outro, além de identificar erros de classificação dos óbitos que modificam os indicadores de mortalidade infantil ${ }^{6,17,18}$. Dessa forma, se configura como excelente ferramenta para melhora da qualidade das informações produzidas além de favorecer a dinâmica dos serviços de vigilância, sobretudo referentes às estatísticas vitais ${ }^{17}$.

\section{Considerações finais}

Dentro do cenário delineado pela literatura analisada neste estudo, é possível traçar algumas considerações sobre a utilização do método de linkage na identificação de fatores de risco associados à mortalidade infantil.
A implantação dos Sistemas de Informação em Saúde brasileiros possibilita um número crescente de estudos com custos reduzidos em sua realização, entretanto, devido às grandes diferenças regionais do Brasil, é necessário considerar a confiabilidade e a completitude do sistema em questão.

Importante estimular a utilização dos dados gerados pelos sistemas de informações de base epidemiológica no planejamento das ações de saúde e a sensibilização dos profissionais para o correto preenchimento dos documentos que os alimentam, no caso específico a Declaração de Óbito e a Declaração de Nascido Vivo.

A oportunidade de analisar informações referentes aos nascimentos e óbitos infantis do SINASC e do SIM favorece novas possibilidades de identificação de fatores determinantes da mortalidade infantil a fim de subsidiar a verificação dos serviços e a organização da rede assistencial.

A utilização da técnica de linkage é de grande valia, possibilitando resgatar na declaração de nascido vivo as variáveis preditoras da mortalidade infantil, viabilizando a pesquisa. O linkage entre SIM e SINASC permite não só maior aproveitamento de sistemas de informações já estruturados nos serviços, como também a formulação de críticas à qualidade desses bancos, as quais podem servir de estímulo para o seu aperfeiçoamento.

Apesar dos fatores que levam ao óbito infantil serem variados e interatuarem entre si com diferentes intensidades, é necessária a adoção de medidas mais diretas nos serviços de saúde e também na comunidade, de modo que possam garantir um atendimento adequado ao binômio mãe-filho no pré-natal, no parto e em todo período neonatal a nível local. 


\section{Colaboradores}

SL Diogenes Santos trabalhou na concepção e no delineamento do estudo, na pesquisa e no levantamento de material bibliográfico, na análise dos dados, na redação e revisão crítica do artigo. ARV Silva trabalhou na concepção e delineamento do estudo e na revisão crítica do artigo. V Campelo trabalhou na revisão crítica do artigo. FT Rodrigues trabalhou na pesquisa e no levantamento de material bibliográfico. JF Ribeiro trabalhou na pesquisa e no levantamento de material bibliográfico.

\section{Referências}

1. Geib LTC, Freu CM, Brandão M, Nunes M L. Determinantes sociais e biológicos da mortalidade infantil em coorte de base populacional em Passo Fundo, Rio Grande do Sul. Cien Saude Colet 2010; 15(2):363-370.

2. Santos HG, Andrade SM, Silva AMR, Carvalho WO, Mesas AE. Risk factors for infant mortality in a municipality in southern Brazil: a comparison of two cohorts using hierarchical analysis. Cad Saude Publica 2012; 28(10):1915-1926.

3. Nascimento RM, Leite AJM, Almeida NMGS, Al meida PC, Silva CF. Determinantes da mortalidade neonatal: estudo caso-controle em Fortaleza, Ceará, Brasil. Cad Saude Publica 2012; 28(3):559-572.

4. Kassar SB, Melo AMC, Coutinho SB, Lima MC, Lira PIC. Determinants of neonatal death with emphasis on health care during pregnancy, childbirth and reproductive history. J Pediatr 2013; 89(3):269-277.

5. Silva CMCD, Gomes KRO, Rocha OAMS, Almeida IMLM, Moita Neto JM. Validade, confiabilidade e evitabilidade da causa básica dos óbitos neonatais ocorridos em unidade de cuidados intensivos da Rede Norte-Nordeste de Saúde Perinatal. Cad Saude Publica 2013; 29(3):547-556.

6. Maia LTS, Souza WV, Mendes ACG. Diferenciais nos fatores de risco para a mortalidade infantil em cinco cidades brasileiras: um estudo de caso-controle com base no SIM e no SINASC. Cad Saude Publica 2012; 28(11):2163-2176.

7. Mombelli MA, Sass A, Molena CAF, Téston EF, Marcon SS. Fatores de risco para mortalidade infantil em municípios do Estado do Paraná, de 1997 a 2008. Rev. Paul. Pediatr. 2012; 30(2):187-194.

8. Lima EFA, Sousa AI, Griep RH, Primo CC. Fatores de risco para mortalidade neonatal no município de Serra, Espírito Santo. Rev. Bras. Enferm 2012; 65(4): 578-585.

9. Mendes KDS, Silveira RCCP, Galvão CM. Revisão integrativa: método de pesquisa para a incorporação de evidências na saúde e na enfermagem. Texto Contexto - Enferm 2008; 17(4):758-764.

10. Rogéria R, Oliveira C, Silva DKF, Bonfim C. Mortalidade neonatal e evitabilidade: uma análise do perfil epidemiológico. Rev. Enferm. UERJ 2011; 19(1):114120.

11. Ferrari RAP, Bertolozzi MR. Mortalidade pós-neonatal no território brasileiro: uma revisão da literatura. Rev, Esc. Enferm. USP 2012; 46(5):1207-1214.

12. Nascimento EMR, Costa MCN, Mota ELA, Paim JS Estudo de fatores de risco para óbitos de menores de um ano mediante compartilhamento de bancos de dados. Cad Saude Publica 2008; 24(11):2593-2602.

13. Soares ES, Menezes GMS. Fatores associados à mortalidade neonatal precoce: análise de situação no nível local. Epidemiol Serv Saúde 2010; 19(1):51-60.

14. Doldan RV, Costa JSD, Nunes MF. Fatores associados à mortalidade infantil no município de Foz do Iguaçu, Paraná, Brasil: estudo de caso-controle. Epidemiol Serv Saúde 2011; 20(4):491-498. 
15. Ferreira JSA, Vilela MBR, Aragão PS, Oliveira RA, Tiné RF. Avaliação da qualidade da informação: linkage entre SIM e SINASC em Jaboatão dos Guararapes (PE). Cien Saude Colet 2011; 16(Supl. 1):12411246.

16. Pedraza DF. Qualidade do Sistema de Informações sobre Nascidos Vivos (Sinasc): análise crítica da literatura. Cien Saude Colet 2012; 17(10):2729-2737.

17. Mendes ACG, Lima MM, Sá DA, Oliveira LCS, Maia LTS. Uso da metodologia de relacionamento de bases de dados para qualificação da informação sobre mortalidade infantil nos municípios de Pernambuco. Rev. Bras. Saude Mater. Infant. 2012; 12(3): 243-249.

18. Figueiroa BQ, Vanderlei LCM, Frias PG, Carvalho PI, Szwarcwald CL. Análise da cobertura do Sistema de Informações sobre Mortalidade em Olinda, Pernambuco, Brasil. Cad Saude Publica 2013; 29(3): 475-484.

Artigo apresentado em 27/08/2013

Aprovado em 27/10/2013

Versão final apresentada em 30/10/2013 